\title{
Feature Extraction of ROI on Image
}

\author{
Zhenyu Han ${ }^{1, ~ a ~}$, Jihong Wang ${ }^{2, b}$, Tianshe Yang ${ }^{3, c}$ and QingE $W^{1, d, ~ * ~}$ \\ ${ }^{1}$ College of Electric and Information Engineering, Zhengzhou University of Light Industry, Zhengzhou, \\ 450002, China \\ ${ }^{2}$ School of Electrical Engineering, zhengzhou University of Science \& Technology, China \\ ${ }^{3}$ China Xi'an Satellite Control Center \\ a331010283@qq.com, b2535703009@qq.com, cyangtianshe001@163.com, d*wqe969699@163.co \\ $\mathrm{m}$, ${ }^{*}$ corresponding author
}

Keywords: Feature extraction; Region of Interest(ROI); Wavelet transform.

\begin{abstract}
This paper presents an algorithm of image segmentation of region of interest (ROI), extracts the ROI of a palmprint image and studies the composing features of palmprint. This paper constructs a coordinates by making use of characteristic points in the palm geometric contour, improves the algorithm of ROI extraction, and provides a positioning method of ROI. Moreover, this paper uses the wavelet transform to divide up ROI, extracts the energy feature of wavelet.
\end{abstract}

\section{Introduction}

Although the research of palmprint recognition started relatively late, it has also experienced stages of emergence, development and maturation during its development of more than ten years. Now it is experiencing the deeper and more detailed development.

In 1985, Matsumoto Katsuhei introduced the application of palmprint recognition in the field of identification firstly, however, without deeper research. In 2004 and 2006, Kong and Zhang D improved the Palmcode method by using 2-dimension Gabor filters[1], and formed a new kind of palmprint feature code called Fusioncode. However, both Palmcode and Fusioncode do not contain the direction information of every point in palmprint, which reduces their distinguishing performance. Additionally, some fruitful researches had been implemented by many universities and institutes in the world.

Among algorithms of image segmentation[2-6], the one of algorithms was Fuzzy C-Means clustering[4] has some better features which can meet the human cognition pattern, be described concisely and clearly, be easy to implement and so on. However, this algorithm has some disadvantages such as its performance depends on the initial clustering center, poor antinoise capability, and slow convergence and so on. From the research of traditional Markov Random Field (MRF) known, the segmentation effect of MRF to micro texture was better, but the segmentation result to macro texture had many isolated islands or small areas.

\section{Extraction of ROI}

In a gathered palmprint image, besides the noise in the data of palm and background, the translation and rotation of palm can influence the quality of image. All these factors do not make against the extraction and matching of palmprint features. Therefore, some preprocessing such as segmentation, calibration and normalization must be implemented before the features extraction of palmprint is carried out.

(1) Extraction of palmprint edge contour

The image border is the discontinuous reflection of local characteristics of an image, such as the mutation of gray level, colors and texture, which marks the end of a region and the beginning of another region. For the calculation simplicity, the first order derivative or the second derivative is 
usually used to check the border of an image. It can be easy to detect the discontinuity of gray level by taking advantage of the derivative. The detection of border can be realized by the convolution based on the spatial differential operators. Some smoothness and binarization processing should be done before the extraction of border is implemented.

Assume the original palmprint image is $f^{\prime}(x, y)$, the smooth filtering to $f^{\prime}(x, y)$ in a spatial domain can be carried out by using a low-pass filter $H$ and the output image is $g(u, v)$, as shown in equation (1).

$$
g(u, v)=\sum_{x} \sum_{y} f^{\prime}(x, y) H(u-x+1, v-y+1)
$$

A relative complete image of palm edge can be obtained after filtering. Then a binarization processing for image $g(u, v)$ is carried out by choosing a proper threshold value $h$. As shown in equation (2), the processed image is $f(x, y)$.

$$
f(x, y)= \begin{cases}1 & g(u, v)>h \\ 0 & g(u, v) \leq h\end{cases}
$$

Then the edge detection for the binarization image can be carried out in image width and image height directions by making use of gray-level mutation. The algorithm is as follows:

Assume the sizes of image height and image width are $m$ and $n$, respectively. The number of gray-level change, $c_{i}, i=1, \cdots, m$, is sought along the image width direction. According to the experimental result to texture and background regions, the number of gray change of texture region is more than that of background region. Further, according to the change number $c_{i}$, a threshold value $M_{1}$ can be given. For any change number is greater than this threshold, i.e., $c_{i}>M_{1}$, at this time, the height and width are recorded, which represented as $y_{k_{1}}$ and $x_{k_{1}}$, respectively, $k_{1} \in N$, and the set of ordered pairs is acquired as equation (3).

$$
I_{1}=\left\{\left(x_{k_{1}}, y_{k_{1}}\right) \mid c_{i}>M_{1}\right\}
$$

On the other hand, in a given fixed point along image width direction case, i.e., width $=n_{j}$, the number of gray-level change, $d_{j}, j=1, \cdots, n$, is sought along the image height direction. Then according to the change number $d_{j}$, another threshold value $M_{2}$ can be given. Similarly, for any change number is greater than this threshold, i.e., $d_{j}>M_{2}$, at this time, the width and height are recorded, which represented as $x_{k_{2}}$ and $y_{k_{2}}$, respectively, $k_{2} \in N$, and the set of ordered pairs is obtained as equation (4).

$$
I_{2}=\left\{\left(x_{k_{2}}, y_{k_{2}}\right) \mid d_{j}>M_{2}\right\}
$$

According to equation (3) and (4), the set of ordered pairs $I=I_{1} \cup I_{2}=\left\{\left(x_{k}, y_{k}\right), k \in N\right\}$ can be obtained. Based on $\left(x_{k}, y_{k}\right)$, the fitting curve that $x_{k}$ and $y_{k}$ satisfy can be obtained. The edge line of palmprint region can be obtained by carrying out borderline modeling of texture. This algorithm of texture segmentation is called the gray-level mutation algorithm.

(2) Extraction of ROI

How to divide up a palmprint image and get the Region of Interest (ROI) that contains the abundant information and simplicity of operation, this paper gives a fast and convenient approach. That is to say, firstly, in the horizontal direction along $x$ axis, searches a sharp growth value $h_{1}$ and a sharp reduction value $h_{2}$ in the gray-level mutation, then the left boundary $l_{1}$ and right boundary $l_{2}$ of ROI can be obtained. Secondly, in the vertical direction along $y$ axis, searches a sharp growth value $w_{1}$ and a sharp reduction value $w_{2}$ in the gray-level mutation, then the upper boundary $l_{3}$ and lower boundary $l_{4}$ of ROI are obtained. Where, the sharp growth and sharp reduction in the gray-level have something to do with the amount of pixel of edge width and edge height of palmprint, 
as well as the position of edge between fingers and palm, i.e., the mutation case of gray-level is decided by the two factors.

A rectangle is formed by the four straight lines $l_{1} 、 l_{2} 、 l_{3} 、 l_{4}$, as shown in Figure 1 . The area of image falling within this rectangle is then extracted as the ROI, as shown in Figure 2. The ROI obtained in this way has eliminated the influence of noise, rotation, translation and so on. Experiment proved that the ROI extracted in this way is more beneficial to the subsequent feature extraction of palmprint.

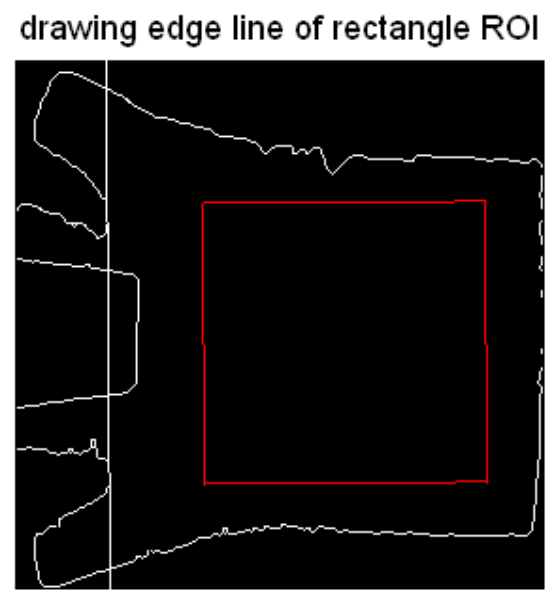

Fig.1. Information-rich rectangle ROI achieved palmprint in rectangle region palmprint extracted in rectangle region
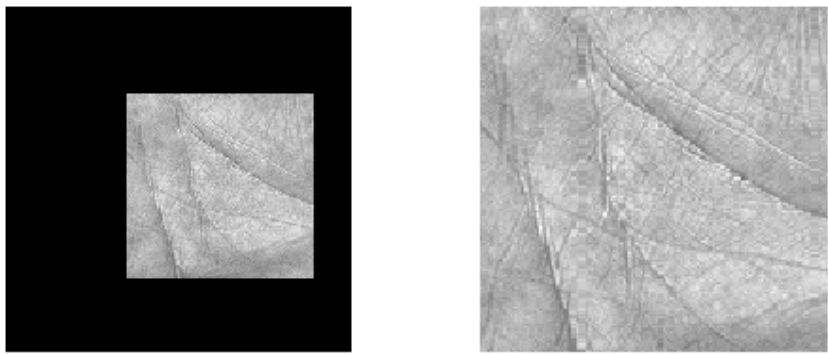

Fig.2. Palmprint extracted in ROI

\section{Feature extraction of ROI}

Here two algorithms on feature extraction are given. The first one is: the gray-level mutation algorithm mentioned above not only can carry out the edge detection of image, but also can implement the characteristic points detection such as corner points, end points, bifurcation points and so on. To acquire these characteristic points, the numbers of gray-level change $c_{i}$ and $d_{j}$ are searched along the image width and image height direction of a palmprint, respectively. If there is $c_{i}>M_{1}$ or $d_{j}>M_{2}$, the detected pixel points are the characteristic points such as edge points, corner points, bifurcation points and so on. Where $M_{1}$ and $M_{2}$ are the two given thresholds. So, the characteristic vector that every detected characteristic point is corresponding to is $V=\left[c_{i}, d_{j}\right]$. The detection of characteristic points in ROI by gray-level change is shown in Figure 3. 


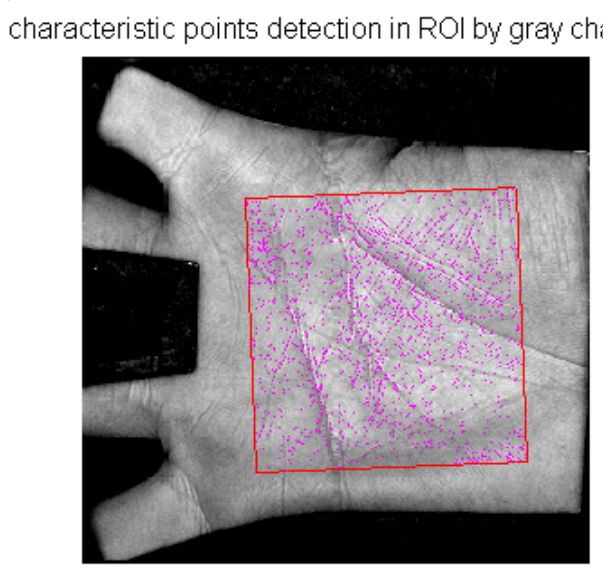

Fig.3. Detection of characteristic points in ROI by gray-level change

The second algorithm is to carry out the feature extraction of palmprint by wavelet transform. Choose a function $\psi(\vec{t})$ to construct $\psi_{\vec{j}, \vec{k}}(\vec{t})=2^{\vec{j} / 2} \psi\left(2^{\vec{j}} \vec{t}-\vec{k}\right)$, and make it do inner product with image $p(x, y)$, which can carry out some processing for a palmprint image such as smoothing, denoising, enhancing, compressing and so on. Where, $\vec{t}=(x, y), \vec{j}=\left(j_{1}, j_{2}\right), \vec{k}=\left(k_{1}, k_{2}\right)$. An alternative way is usually to choose a two-dimension Gabor function as a wavelet as follows:

$$
g(x, y)=\frac{1}{2 \pi \delta_{x} \delta_{y}} \cdot e^{-\frac{1}{2}\left[\left(\frac{x}{\delta_{x}}\right)^{2}+\left(\frac{y}{\delta_{y}}\right)^{2}\right]+j(u x+v y)}
$$

Make it process the image $p(x, y)$, then the concrete algorithm is $W_{\vec{j}, \vec{k}}=\left\langle\psi_{\vec{j}, \vec{k}}(\vec{t}), p(x, y)\right\rangle$, which can carry out a decomposition and extraction of energy coefficient features of image $p(x, y)$. For a region of texture with rich features, it shows that the energy is denser, the difference in amplitude is little, and the distribution of energy is regular as the decomposition levels increase. For a region with sparse texture, the energy takes on the form of jumping, sparse rendering as the decomposition levels increase.

From the decomposition calculation of wavelet known, the ith level energy feature of ripple is calculated by the ith level decomposition coefficient of wavelet, which shows the texture feature of a palmprint image in the $2^{-i}$ scale, in different directions and different positions.

The palmprint image passes through $n$-level two-dimensional discrete wavelet transform, the detail image of each level in horizontal $\left(H_{i}\right)$, vertical $\left(V_{i}\right)$ and diagonal $\left(D_{i}\right)$ directions can be obtained, respectively, as shown in Figure 4, where $i=1,2, \cdots, n$.

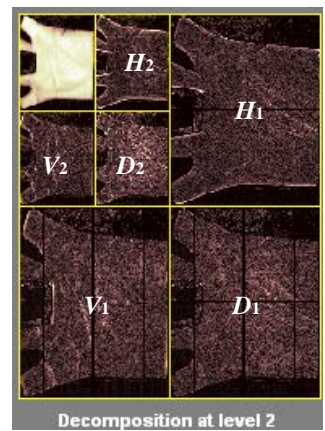

(a) 2-level decomposition

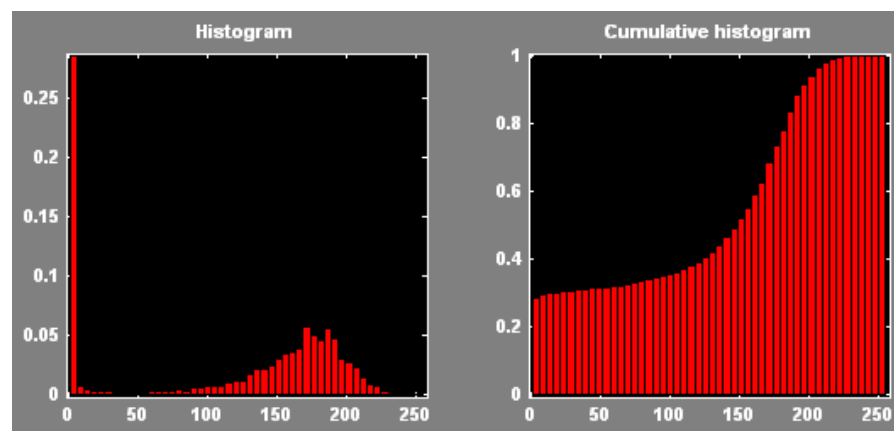

(b) energy histogram

Fig.4. 2-level decomposition and its energy histogram 


\section{Conclusion.}

This paper presents two segmentation algorithms of ROI, which the one is based on the palm edge contour and the other is based on the distance and direction. Moreover, this paper gives the feature extraction algorithm of ROI, and carries out the simulation test. Finally, the simulation results prove the feasibility and effectiveness of all proposed algorithms or approaches in each stage.

\section{Acknowledgments}

This work is supported by Henan Province Outstanding Youth on Science and Technology Innovation; Project of Henan Province Science and Technology (No: 142300410247); Key Project of Henan Province Education Department (No: 14A413002); Project funded by China postdoctoral science foundation (No: 2013M531120); Aerospace support fund (No: Chinare2014-04-07); Project of Zhengzhou Science and Technology Department (No: 131PPTGG411-4); Key Laboratory of Digital Ocean, SOA (KLD0201408); National Natural Science Foundation of China (No. 61501407); Key Project of Henan Institutions of Higher Learning (No. 15A413006), respectively.

\section{References}

[1] Kong W, Zhang D, Kame M, Palmprint identification using feature-level fusion, Pattern recognition. 39 (2006) 478-487.

[2] Xiaozhen Xie, Jitao Wu, Minggang Jing, Fast two-stage segmentation via non-local active contours in multiscale texture feature space, Pattern Recognition Letters. 34 (2013) 1230-1239.

[3] Edward H.S. Lo, Mark R. Pickering, Michael R. Frater, John F. Arnold, Image segmentation from scale and rotation invariant texture features from the double dyadic dual-tree complex wavelet transform, Image and Vision Computing. 29 (2011) 15-28.

[4] Jian Yu, Texture segmentation based on FCM algorithm combined with GLCM and space information, International Conference on Electric Information and Control Engineering. (2011) 4569 $-4572$.

[5] Zhiling Long, Nicolas H. Younan, Multiscale texture segmentation via a contourlet contextual hidden Markov model, Digital Signal Processing. 23 (2013) 859-869.

[6] Nikolaos Dimitriou, Anastasios Delopoulos, Incorporating higher order models for occlusion resilient motion segmentation in streaming videos, Image and Vision Computing,, 36 (2015) 70-82. 